\title{
Synthesis and characterization of new trimeric rhenium(I) complexes. The influence of steric factors on the size of pyrazolonate-rhenium(I) metallomacrocycles.
}

Paula Barbazán, Rosa Carballo, José S. Casas, Emilia García-Martínez, Gumersindo PereirasGabián, Agustín Sánchez, and Ezequiel M. Vázquez-López*

From the Departaments of Inorganic Chemistry of the Universities of Vigo and Santiago de Compostela, Galicia, Spain

\section{Supporting Materials}

\footnotetext{
* To whom correspondence should be addressed at Departamento de Química Inorgánica, Facultade de Química, Edificio de Ciencias Experimentais, Universidade de Vigo, E-36310 Vigo, Galicia, Spain. E-mail: ezequiel@uvigo.es.
} 
Table S1. Crystal and structure refinement data.

2b.1/2 $\mathrm{C}_{7} \mathrm{H}_{\mathbf{8}}$

Empirical formula

Formula weight

Temperature

Wavelength

Crystal system

Space group

Unit cell dimensions

$\mathrm{a}(\AA)$

$\mathrm{b}(\AA)$

$\mathrm{c}(\AA)$

$\beta\left(^{\circ}\right)$

Volume $\left(\AA^{3}\right)$

Z

Density (calculated) Mg/m 3

Absorption coefficient $\left(\mathrm{mm}^{-1}\right)$

Crystal size, $\mathrm{mm}^{3}$

$\theta$ range for data collection $\left(^{\circ}\right)$

Index ranges $(\mathrm{h} ; \mathrm{k} ; \mathrm{l})$

Reflections collected

Independent reflections ( $\left.\mathrm{R}_{\text {int }}\right)$

Absorption correction

Max. and min. transmission

Refinement method

Data / restraints / parameters

Goodness-of-fit on $\mathrm{F}^{2}$

Final R indices [I>2sigma(I)]

$\mathrm{R}$ indices (all data)

Absolute structure parameter Largest diff. peak and hole

9231 (0.0818)

equivalents on $\mathrm{F}^{2}$

9231 / 2 / 455

0.791

0.1525

0.1742

$0.08(2)$ 2c. $2 \mathrm{C}_{7} \mathrm{H}_{8}$

$\mathrm{C}_{56} \mathrm{H}_{46} \mathrm{~N}_{9} \mathrm{O}_{12} \mathrm{~S}_{3} \mathrm{Re}_{3}$

1691.80

293(2) K

$0.71073 \AA$

Monoclinic

P2(1)/C

17.2386(11)

13.0484(8)

27.6698(17)

104.497(2)

6025.8(6)

4

\subsection{5}

6.182

$0.12 \times 0.14 \times 0.17$

$1.52-28.03^{\circ}$

$-22,20:-16,17 ;-32,36$

32147

13329 (0.0904)

Semi-empirical

from

1.000000 and 0.809091

Full-matrix least-squares

$\mathrm{R} 1=0.0700, \quad \mathrm{wR} 2=$

$\mathrm{R} 1=0.1252, \quad \mathrm{wR} 2=$

1.518 and -1.585 e. $\AA^{-3}$
Semi-empirical from

equivalents

1.000000 and 0.888868

Full-matrix least-

squares on $\mathrm{F}^{2}$

13329 / 0 / 638

0.691

$\mathrm{R} 1=0.0446, \quad \mathrm{wR} 2=$ 0.0596

$\mathrm{R} 1=0.2007, \quad \mathrm{wR} 2=$

0.0801

0.901 and -0.792 e. $\AA^{-3}$ 2c

$\mathrm{C}_{42} \mathrm{H}_{30} \mathrm{~N}_{9} \mathrm{O}_{12} \mathrm{~S}_{3} \mathrm{Re}_{3}$

1507.53

293(2) K

$0.71073 \AA$

Monoclinic

P2(1)/n

19.0752(15)

16.8519(13)

19.3670(15)

$112.146(2)^{\circ}$

5766.3(8) $\AA^{3}$

4

1.737

6.449

$0.34 \times 0.14 \times 0.13$

1.66-28.05․

$-24,24 ;-20,22 ;-24,25$

31573

12926 (0.1038)

from equivalents

1.000000 and 0.502199

Full-matrix least-

squares on $\mathrm{F}^{2}$

12926 / 0 / 622

0.852

$\mathrm{R} 1=0.0610, \mathrm{wR} 2=$ 0.1364

$\mathrm{R} 1=0.1714, \mathrm{wR} 2=$ 0.1591

1.851 and -1.698 e. $\AA^{-3}$ 
Table S2. Bond lengths $[\AA]$ and angles $\left[^{\circ}\right]$ in $\mathbf{2 b} \cdot \mathbf{1} / \mathbf{2} \mathbf{C}_{\mathbf{7}} \mathbf{H}_{8}$.

\begin{tabular}{|c|c|c|c|c|c|}
\hline $\operatorname{Re}(1)-C(121)$ & $1.88(3)$ & $\mathrm{C}(12)-\mathrm{C}(13)$ & $1.37(4)$ & $\mathrm{C}(22)-\mathrm{C}(25)$ & $1.44(3)$ \\
\hline $\operatorname{Re}(1)-C(123)$ & 1.92(3) & C(13)-C(14) & 1.33(3) & C(23)-C(24) & $1.48(4)$ \\
\hline $\operatorname{Re}(1)-C(122)$ & $1.93(4)$ & $\operatorname{Re}(2)-C(222)$ & $1.85(3)$ & $\operatorname{Re}(3)-C(321)$ & $1.520(6)$ \\
\hline $\mathrm{Re}(1)-\mathrm{O}(21)$ & $2.143(17)$ & $\operatorname{Re}(2)-C(221)$ & $1.92(3)$ & $\operatorname{Re}(3)-C(323)$ & $1.84(5)$ \\
\hline $\operatorname{Re}(1)-N(13)$ & $2.15(2)$ & $\operatorname{Re}(2)-C(223)$ & 2.04(3) & $\operatorname{Re}(3)-C(322)$ & $2.093(12)$ \\
\hline $\operatorname{Re}(1)-S(1)$ & $2.454(6)$ & $\operatorname{Re}(2)-\mathrm{N}(23)$ & $2.21(2)$ & Re(3)-N(33) & $2.15(3)$ \\
\hline $\operatorname{Re}(1)-\operatorname{Re}(2)$ & 7.3791(17) & $\operatorname{Re}(2)-O(31)$ & $2.29(2)$ & $\operatorname{Re}(3)-S(3)$ & $2.422(8)$ \\
\hline $\operatorname{Re}(1)-\operatorname{Re}(3)$ & 7.4420(17) & $\operatorname{Re}(2)-S(2)$ & $2.437(8)$ & $\mathrm{C}(321)-\mathrm{O}(321)$ & 1.5470 \\
\hline $\mathrm{C}(121)-\mathrm{O}(121)$ & $1.14(3)$ & $\operatorname{Re}(2)-\operatorname{Re}(3)$ & $7.472(2)$ & $\mathrm{C}(322)-\mathrm{O}(322)$ & $0.92(2)$ \\
\hline $\mathrm{C}(122)-\mathrm{O}(122)$ & $1.13(3)$ & $\mathrm{C}(223)-\mathrm{O}(223)$ & $1.05(3)$ & $\mathrm{C}(323)-\mathrm{O}(323)$ & $1.17(5)$ \\
\hline $\mathrm{C}(123)-\mathrm{O}(123)$ & 1.16(3) & $\mathrm{C}(221)-\mathrm{O}(221)$ & 1.11(3) & $S(3)-C(31)$ & $1.83(4)$ \\
\hline$S(1)-C(11)$ & $1.59(2)$ & $\mathrm{C}(222)-\mathrm{O}(222)$ & $1.25(3)$ & $\mathrm{O}(31)-\mathrm{C}(34)$ & 1.14(3) \\
\hline $\mathrm{O}(11)-\mathrm{C}(14)$ & $1.31(2)$ & $S(2)-C(21)$ & $1.78(3)$ & N(31)-C(31) & $1.17(4)$ \\
\hline $\mathrm{O}(11)-\operatorname{Re}(3)$ & $2.132(18)$ & $\mathrm{O}(21)-\mathrm{C}(24)$ & $1.21(3)$ & N(31)-C(311) & $1.59(3)$ \\
\hline N(11)-C(11) & $1.45(3)$ & N(21)-C(21) & $1.31(4)$ & $\mathrm{C}(31)-\mathrm{N}(32)$ & $1.36(3)$ \\
\hline $\mathrm{N}(11)-\mathrm{C}(111)$ & $1.47(3)$ & $\mathrm{N}(21)-\mathrm{C}(211)$ & $1.45(3)$ & $\mathrm{N}(32)-\mathrm{N}(33)$ & $1.39(3)$ \\
\hline $\mathrm{C}(11)-\mathrm{N}(12)$ & $1.44(3)$ & $\mathrm{C}(21)-\mathrm{N}(22)$ & $1.30(3)$ & $\mathrm{N}(32)-\mathrm{C}(34)$ & $1.44(3)$ \\
\hline $\mathrm{N}(12)-\mathrm{N}(13)$ & $1.38(2)$ & $\mathrm{N}(22)-\mathrm{N}(23)$ & $1.39(3)$ & $\mathrm{N}(33)-\mathrm{C}(32)$ & $1.27(3)$ \\
\hline $\mathrm{N}(12)-\mathrm{C}(14)$ & $1.44(3)$ & $\mathrm{N}(22)-\mathrm{C}(24)$ & $1.51(3)$ & $\mathrm{C}(32)-\mathrm{C}(35)$ & $1.42(3)$ \\
\hline $\mathrm{N}(13)-\mathrm{C}(12)$ & $1.30(3)$ & $\mathrm{N}(23)-\mathrm{C}(22)$ & $1.43(3)$ & C(32)-C(33) & $1.53(4)$ \\
\hline $\mathrm{C}(12)-\mathrm{C}(15)$ & $1.37(3)$ & $\mathrm{C}(22)-\mathrm{C}(23)$ & $1.35(4)$ & C(33)-C(34) & $1.42(4)$ \\
\hline $\mathrm{C}(121)-\operatorname{Re}(1)-\mathrm{C}(123)$ & 89.3(10) & $\mathrm{C}(222)-\operatorname{Re}(2)-\mathrm{C}(221)$ & $88.1(12)$ & $\mathrm{C}(321)-\operatorname{Re}(3)-\mathrm{C}(323)$ & 88.1(16) \\
\hline $\mathrm{C}(121)-\operatorname{Re}(1)-\mathrm{C}(122)$ & $86.3(11)$ & $C(222)-\operatorname{Re}(2)-C(223)$ & $90.0(11)$ & $\mathrm{C}(321)-\operatorname{Re}(3)-\mathrm{C}(322)$ & $91.4(6)$ \\
\hline $\mathrm{C}(123)-\operatorname{Re}(1)-\mathrm{C}(122)$ & $90.6(11)$ & $\mathrm{C}(221)-\operatorname{Re}(2)-\mathrm{C}(223)$ & $87.3(12)$ & $\mathrm{C}(323)-\operatorname{Re}(3)-\mathrm{C}(322)$ & $92.8(17)$ \\
\hline $\mathrm{C}(121)-\operatorname{Re}(1)-\mathrm{O}(21)$ & $97.2(9)$ & $\mathrm{C}(222)-\operatorname{Re}(2)-\mathrm{N}(23)$ & $171.9(11)$ & $\mathrm{C}(321)-\mathrm{Re}(3)-\mathrm{O}(11)$ & $98.9(5)$ \\
\hline $\mathrm{C}(123)-\operatorname{Re}(1)-\mathrm{O}(21)$ & 173.3(7) & $\mathrm{C}(221)-\mathrm{Re}(2)-\mathrm{N}(23)$ & $98.8(10)$ & $\mathrm{C}(323)-\mathrm{Re}(3)-\mathrm{O}(11)$ & $172.6(17)$ \\
\hline $\mathrm{C}(122)-\mathrm{Re}(1)-\mathrm{O}(21)$ & $91.3(10)$ & $\mathrm{C}(223)-\operatorname{Re}(2)-\mathrm{N}(23)$ & $94.7(10)$ & $\mathrm{C}(322)-\mathrm{Re}(3)-\mathrm{O}(11)$ & $89.6(5)$ \\
\hline $\mathrm{C}(121)-\operatorname{Re}(1)-\mathrm{N}(13)$ & $98.3(10)$ & $\mathrm{C}(222)-\mathrm{Re}(2)-\mathrm{O}(31)$ & $90.5(10)$ & C(321)-Re(3)-N(33) & $95.6(8)$ \\
\hline $\mathrm{C}(123)-\mathrm{Re}(1)-\mathrm{N}(13)$ & $97.2(10)$ & $\mathrm{C}(221)-\mathrm{Re}(2)-\mathrm{O}(31)$ & $97.8(12)$ & C(323)-Re(3)-N(33) & $96.6(18)$ \\
\hline $\mathrm{C}(122)-\operatorname{Re}(1)-\mathrm{N}(13)$ & $170.9(9)$ & $\mathrm{C}(223)-\mathrm{Re}(2)-\mathrm{O}(31)$ & $174.9(11)$ & $\mathrm{C}(322)-\mathrm{Re}(3)-\mathrm{N}(33)$ & $168.5(6)$ \\
\hline $\mathrm{O}(21)-\mathrm{Re}(1)-\mathrm{N}(13)$ & $80.4(8)$ & $\mathrm{N}(23)-\mathrm{Re}(2)-\mathrm{O}(31)$ & $84.3(9)$ & $\mathrm{O}(11)-\mathrm{Re}(3)-\mathrm{N}(33)$ & $80.3(8)$ \\
\hline C(121)-Re(1)-S(1) & $177.4(8)$ & $C(222)-\operatorname{Re}(2)-S(2)$ & $94.6(9)$ & C(321)-Re(3)-S(3) & $175.9(5)$ \\
\hline$C(123)-\operatorname{Re}(1)-S(1)$ & $93.1(6)$ & $C(221)-\operatorname{Re}(2)-S(2)$ & 177.1(8) & $C(323)-\operatorname{Re}(3)-S(3)$ & $94.3(16)$ \\
\hline $\mathrm{C}(122)-\operatorname{Re}(1)-\mathrm{S}(1)$ & $94.7(7)$ & $C(223)-\operatorname{Re}(2)-S(2)$ & $94.0(8)$ & $\mathrm{C}(322)-\operatorname{Re}(3)-\mathrm{S}(3)$ & $91.8(3)$ \\
\hline $\mathrm{O}(21)-\operatorname{Re}(1)-\mathrm{S}(1)$ & $80.3(4)$ & $N(23)-\operatorname{Re}(2)-S(2)$ & $78.5(6)$ & $\mathrm{O}(11)-\operatorname{Re}(3)-\mathrm{S}(3)$ & $78.5(5)$ \\
\hline N(13)-Re(1)-S(1) & $80.4(6)$ & $\mathrm{O}(31)-\operatorname{Re}(2)-\mathrm{S}(2)$ & $80.9(8)$ & N(33)-Re(3)-S(3) & $80.8(7)$ \\
\hline $\mathrm{O}(121)-\mathrm{C}(121)-\operatorname{Re}(1)$ & $176(3)$ & $\mathrm{O}(223)-\mathrm{C}(223)-\mathrm{Re}(2)$ & $175(3)$ & $\operatorname{Re}(3)-\mathrm{C}(321)-\mathrm{O}(321)$ & $172.3(4)$ \\
\hline $\mathrm{O}(122)-\mathrm{C}(122)-\operatorname{Re}(1)$ & $175(2)$ & $\mathrm{O}(221)-\mathrm{C}(221)-\mathrm{Re}(2)$ & 173(3) & $\mathrm{O}(322)-\mathrm{C}(322)-\operatorname{Re}(3)$ & $165.5(18)$ \\
\hline $\mathrm{O}(123)-\mathrm{C}(123)-\operatorname{Re}(1)$ & $177(2)$ & $\mathrm{O}(222)-\mathrm{C}(222)-\mathrm{Re}(2)$ & $174(2)$ & $\mathrm{O}(323)-\mathrm{C}(323)-\operatorname{Re}(3)$ & $176(5)$ \\
\hline $\mathrm{C}(11)-\mathrm{S}(1)-\operatorname{Re}(1)$ & $97.2(10)$ & $C(21)-S(2)-\operatorname{Re}(2)$ & $99.4(10)$ & $\mathrm{C}(31)-\mathrm{S}(3)-\operatorname{Re}(3)$ & $96.7(9)$ \\
\hline $\mathrm{C}(14)-\mathrm{O}(11)-\operatorname{Re}(3)$ & $128.9(18)$ & $\mathrm{C}(24)-\mathrm{O}(21)-\mathrm{Re}(1)$ & $131.2(18)$ & $\mathrm{C}(34)-\mathrm{O}(31)-\mathrm{Re}(2)$ & $131(2)$ \\
\hline $\mathrm{C}(11)-\mathrm{N}(11)-\mathrm{C}(111)$ & $117(2)$ & $\mathrm{C}(21)-\mathrm{N}(21)-\mathrm{C}(211)$ & $127(3)$ & $\mathrm{C}(31)-\mathrm{N}(31)-\mathrm{C}(311)$ & $129(4)$ \\
\hline $\mathrm{N}(12)-\mathrm{C}(11)-\mathrm{N}(11)$ & $108.8(19)$ & $\mathrm{N}(22)-\mathrm{C}(21)-\mathrm{N}(21)$ & 124(3) & $\mathrm{N}(31)-\mathrm{C}(31)-\mathrm{N}(32)$ & 119(3) \\
\hline $\mathrm{N}(12)-\mathrm{C}(11)-\mathrm{S}(1)$ & $126.9(18)$ & $N(22)-C(21)-S(2)$ & 117(3) & $N(31)-C(31)-S(3)$ & 113(3) \\
\hline $\mathrm{N}(11)-\mathrm{C}(11)-\mathrm{S}(1)$ & $124.3(19)$ & $\mathrm{N}(21)-\mathrm{C}(21)-\mathrm{S}(2)$ & 119(2) & N(32)-C(31)-S(3) & $114(3)$ \\
\hline $\mathrm{N}(13)-\mathrm{N}(12)-\mathrm{C}(11)$ & $115.2(19)$ & $\mathrm{C}(21)-\mathrm{N}(22)-\mathrm{N}(23)$ & 125(3) & $\mathrm{C}(31)-\mathrm{N}(32)-\mathrm{N}(33)$ & 122(3) \\
\hline $\mathrm{N}(13)-\mathrm{N}(12)-\mathrm{C}(14)$ & $113(2)$ & $\mathrm{C}(21)-\mathrm{N}(22)-\mathrm{C}(24)$ & $124(2)$ & $\mathrm{C}(31)-\mathrm{N}(32)-\mathrm{C}(34)$ & $124(3)$ \\
\hline $\mathrm{C}(11)-\mathrm{N}(12)-\mathrm{C}(14)$ & $131.8(19)$ & $\mathrm{N}(23)-\mathrm{N}(22)-\mathrm{C}(24)$ & $108.3(19)$ & $\mathrm{N}(33)-\mathrm{N}(32)-\mathrm{C}(34)$ & $112(2)$ \\
\hline $\mathrm{C}(12)-\mathrm{N}(13)-\mathrm{N}(12)$ & 102.2(19) & $\mathrm{N}(22)-\mathrm{N}(23)-\mathrm{C}(22)$ & $110(2)$ & $\mathrm{C}(32)-\mathrm{N}(33)-\mathrm{N}(32)$ & $113(3)$ \\
\hline $\mathrm{C}(12)-\mathrm{N}(13)-\mathrm{Re}(1)$ & $138.9(17)$ & $\mathrm{N}(22)-\mathrm{N}(23)-\mathrm{Re}(2)$ & $112.8(14)$ & $\mathrm{C}(32)-\mathrm{N}(33)-\operatorname{Re}(3)$ & $129(2)$ \\
\hline $\mathrm{N}(12)-\mathrm{N}(13)-\mathrm{Re}(1)$ & $118.7(16)$ & $\mathrm{C}(22)-\mathrm{N}(23)-\operatorname{Re}(2)$ & 134.8(19) & $\mathrm{N}(32)-\mathrm{N}(33)-\mathrm{Re}(3)$ & $117.6(18)$ \\
\hline $\mathrm{N}(13)-\mathrm{C}(12)-\mathrm{C}(15)$ & $119(2)$ & $\mathrm{C}(23)-\mathrm{C}(22)-\mathrm{N}(23)$ & $108(3)$ & $\mathrm{N}(33)-\mathrm{C}(32)-\mathrm{C}(35)$ & $137(3)$ \\
\hline $\mathrm{N}(13)-\mathrm{C}(12)-\mathrm{C}(13)$ & $113(2)$ & $C(23)-C(22)-C(25)$ & 132(2) & $\mathrm{N}(33)-\mathrm{C}(32)-\mathrm{C}(33)$ & $104(2)$ \\
\hline $\mathrm{C}(15)-\mathrm{C}(12)-\mathrm{C}(13)$ & $127(2)$ & $N(23)-C(22)-C(25)$ & $120(2)$ & $C(35)-C(32)-C(33)$ & $115(2)$ \\
\hline$C(14)-C(13)-C(12)$ & $110(3)$ & $C(22)-C(23)-C(24)$ & 112(3) & $C(34)-C(33)-C(32)$ & $110(2)$ \\
\hline $\mathrm{O}(11)-\mathrm{C}(14)-\mathrm{C}(13)$ & $140(3)$ & $\mathrm{O}(21)-\mathrm{C}(24)-\mathrm{C}(23)$ & 140(3) & $\mathrm{O}(31)-\mathrm{C}(34)-\mathrm{C}(33)$ & 144(3) \\
\hline $\mathrm{O}(11)-\mathrm{C}(14)-\mathrm{N}(12)$ & $118(2)$ & $\mathrm{O}(21)-\mathrm{C}(24)-\mathrm{N}(22)$ & $118(2)$ & $\mathrm{O}(31)-\mathrm{C}(34)-\mathrm{N}(32)$ & $116(3)$ \\
\hline $\mathrm{C}(13)-\mathrm{C}(14)-\mathrm{N}(12)$ & $101(2)$ & $\mathrm{C}(23)-\mathrm{C}(24)-\mathrm{N}(22)$ & $101(2)$ & $\mathrm{C}(33)-\mathrm{C}(34)-\mathrm{N}(32)$ & $100(2)$ \\
\hline
\end{tabular}


Table S3. Bond lengths $[\AA]]$ and angles $\left[^{\circ}\right]$ in $\mathbf{2} \mathbf{c} . \mathbf{C}_{\mathbf{7}} \mathbf{H}_{8}$.

\begin{tabular}{|c|c|c|c|c|c|}
\hline $\operatorname{Re}(1)-C(121)$ & $1.860(12)$ & $\mathrm{Re}(2)-\mathrm{O}(31)$ & $2.169(7)$ & $S(3)-C(31)$ & $1.697(11)$ \\
\hline $\operatorname{Re}(1)-C(123)$ & $1.877(13)$ & $\operatorname{Re}(2)-N(23)$ & $2.170(8)$ & N(31)-C(31) & $1.336(11)$ \\
\hline $\operatorname{Re}(1)-C(122)$ & $1.919(11)$ & $\operatorname{Re}(2)-S(2)$ & $2.470(3)$ & N(31)-C(311) & $1.415(12)$ \\
\hline $\operatorname{Re}(1)-N(13)$ & $2.173(9)$ & $\operatorname{Re}(2)-\operatorname{Re}(3)$ & 7.4696(7) & N(32)-C(31) & $1.331(11)$ \\
\hline $\mathrm{Re}(1)-\mathrm{O}(21)$ & $2.173(6)$ & $\mathrm{C}(221)-\mathrm{O}(221)$ & $1.197(11)$ & N(32)-N(33) & $1.386(10)$ \\
\hline $\operatorname{Re}(1)-S(1)$ & 2.461(3) & $\mathrm{C}(222)-\mathrm{O}(222)$ & $1.186(12)$ & N(32)-C(34) & $1.416(12)$ \\
\hline $\operatorname{Re}(1)-\operatorname{Re}(2)$ & $7.4368(8)$ & $\mathrm{C}(223)-\mathrm{O}(223)$ & $1.196(13)$ & N(33)-C(32) & $1.314(12)$ \\
\hline $\operatorname{Re}(1)-\operatorname{Re}(3)$ & $7.4630(7)$ & $S(2)-C(21)$ & $1.684(10)$ & $\mathrm{O}(31)-\mathrm{C}(34)$ & $1.283(11)$ \\
\hline $\mathrm{C}(121)-\mathrm{O}(121)$ & $1.195(11)$ & $\mathrm{N}(21)-\mathrm{C}(21)$ & $1.340(11)$ & $\mathrm{C}(32)-\mathrm{C}(33)$ & $1.386(12)$ \\
\hline $\mathrm{C}(122)-\mathrm{O}(122)$ & $1.164(10)$ & $\mathrm{N}(21)-\mathrm{C}(211)$ & $1.429(12)$ & C(32)-C(35) & $1.515(13)$ \\
\hline $\mathrm{C}(123)-\mathrm{O}(123)$ & $1.151(12)$ & $\mathrm{N}(22)-\mathrm{C}(21)$ & $1.389(11)$ & C(33)-C(34) & $1.370(13)$ \\
\hline$S(1)-C(11)$ & $1.662(10)$ & $\mathrm{N}(22)-\mathrm{N}(23)$ & $1.389(9)$ & $\mathrm{C}(311)-\mathrm{C}(316)$ & $1.310(13)$ \\
\hline $\mathrm{O}(11)-\mathrm{C}(14)$ & $1.276(11)$ & $\mathrm{N}(22)-\mathrm{C}(24)$ & $1.409(11)$ & $\mathrm{C}(311)-\mathrm{C}(312)$ & $1.333(15)$ \\
\hline $\mathrm{O}(11)-\operatorname{Re}(3)$ & $2.205(7)$ & $\mathrm{N}(23)-\mathrm{C}(22)$ & $1.342(11)$ & $\mathrm{C}(312)-\mathrm{C}(313)$ & $1.427(18)$ \\
\hline $\mathrm{N}(11)-\mathrm{C}(11)$ & $1.310(10)$ & $\mathrm{O}(21)-\mathrm{C}(24)$ & $1.304(11)$ & $\mathrm{C}(313)-\mathrm{C}(314)$ & $1.313(18)$ \\
\hline $\mathrm{N}(11)-\mathrm{C}(111)$ & $1.440(11)$ & $\mathrm{C}(22)-\mathrm{C}(23)$ & $1.382(13)$ & $\mathrm{C}(314)-\mathrm{C}(315)$ & $1.324(18)$ \\
\hline $\mathrm{N}(12)-\mathrm{C}(11)$ & $1.393(11)$ & $\mathrm{C}(22)-\mathrm{C}(25)$ & $1.484(12)$ & $C(315)-C(316)$ & $1.399(16)$ \\
\hline $\mathrm{N}(12)-\mathrm{N}(13)$ & $1.411(10)$ & $\mathrm{C}(23)-\mathrm{C}(24)$ & $1.356(13)$ & $C(411)-C(417)$ & $1.311(8)$ \\
\hline $\mathrm{N}(12)-\mathrm{C}(14)$ & $1.444(11)$ & $C(211)-C(216)$ & $1.363(13)$ & $C(411)-C(416)$ & 1.3919 \\
\hline $\mathrm{N}(13)-\mathrm{C}(12)$ & $1.321(11)$ & $C(211)-C(212)$ & $1.375(14)$ & $C(411)-C(412)$ & 1.4838 \\
\hline $\mathrm{C}(12)-\mathrm{C}(13)$ & $1.401(12)$ & $C(212)-C(213)$ & $1.404(15)$ & $C(412)-C(413)$ & 1.4851 \\
\hline $\mathrm{C}(12)-\mathrm{C}(15)$ & $1.483(12)$ & $C(213)-C(214)$ & $1.386(16)$ & $C(413)-C(414)$ & 1.4470 \\
\hline $\mathrm{C}(13)-\mathrm{C}(14)$ & $1.411(13)$ & $C(214)-C(215)$ & $1.370(17)$ & $C(414)-C(415)$ & 1.5308 \\
\hline $\mathrm{C}(111)-\mathrm{C}(112)$ & $1.338(13)$ & $C(215)-C(216)$ & $1.353(14)$ & $C(415)-C(416)$ & 1.2262 \\
\hline$C(111)-C(116)$ & $1.367(14)$ & $\operatorname{Re}(3)-C(321)$ & $1.833(14)$ & $\mathrm{C}(511)-\mathrm{C}(517)$ & $1.364(15)$ \\
\hline$C(112)-C(113)$ & $1.398(14)$ & $\operatorname{Re}(3)-C(322)$ & $1.898(11)$ & $\mathrm{C}(511)-\mathrm{C}(512)$ & 1.3900 \\
\hline $\mathrm{C}(113)-\mathrm{C}(114)$ & $1.351(15)$ & $\operatorname{Re}(3)-C(323)$ & $1.925(12)$ & $C(511)-C(516)$ & 1.3900 \\
\hline$C(114)-C(115)$ & $1.321(15)$ & $\operatorname{Re}(3)-N(33)$ & $2.143(8)$ & $C(512)-C(513)$ & 1.3900 \\
\hline$C(115)-C(116)$ & $1.406(13)$ & $\operatorname{Re}(3)-S(3)$ & $2.461(3)$ & $\mathrm{C}(513)-\mathrm{C}(514)$ & 1.3900 \\
\hline $\operatorname{Re}(2)-C(221)$ & $1.837(12)$ & $\mathrm{O}(321)-\mathrm{C}(321)$ & $1.201(12)$ & $C(514)-C(515)$ & 1.3900 \\
\hline $\operatorname{Re}(2)-C(223)$ & $1.875(14)$ & $\mathrm{O}(322)-\mathrm{C}(322)$ & $1.157(10)$ & $\mathrm{C}(515)-\mathrm{C}(516)$ & 1.3900 \\
\hline $\operatorname{Re}(2)-C(222)$ & $1.882(13)$ & $\mathrm{O}(323)-\mathrm{C}(323)$ & $1.137(11)$ & & \\
\hline C(121)-Re(1)-C(123) & $87.5(5)$ & $\mathrm{C}(222)-\mathrm{Re}(2)-\mathrm{O}(31)$ & $96.7(4)$ & $\mathrm{N}(33)-\operatorname{Re}(3)-\mathrm{O}(11)$ & $81.9(3)$ \\
\hline $\mathrm{C}(121)-\operatorname{Re}(1)-\mathrm{C}(122)$ & $87.5(4)$ & $\mathrm{C}(221)-\operatorname{Re}(2)-\mathrm{N}(23)$ & $98.2(4)$ & C(321)-Re(3)-S(3) & $98.5(4)$ \\
\hline C(123)-Re(1)-C(122) & $87.7(4)$ & $\mathrm{C}(223)-\operatorname{Re}(2)-\mathrm{N}(23)$ & $171.6(5)$ & C(322)-Re(3)-S(3) & $93.3(3)$ \\
\hline $\mathrm{C}(121)-\operatorname{Re}(1)-\mathrm{N}(13)$ & $99.9(4)$ & $\mathrm{C}(222)-\mathrm{Re}(2)-\mathrm{N}(23)$ & $99.3(4)$ & $\mathrm{C}(323)-\operatorname{Re}(3)-S(3)$ & $173.2(4)$ \\
\hline C(123)-Re(1)-N(13) & $97.7(4)$ & $\mathrm{O}(31)-\mathrm{Re}(2)-\mathrm{N}(23)$ & $80.4(3)$ & N(33)-Re(3)-S(3) & 78.5(3) \\
\hline C(122)-Re(1)-N(13) & $171.0(4)$ & $\mathrm{C}(221)-\operatorname{Re}(2)-\mathrm{S}(2)$ & $94.6(4)$ & $\mathrm{O}(11)-\operatorname{Re}(3)-\mathrm{S}(3)$ & $80.06(19)$ \\
\hline $\mathrm{C}(121)-\operatorname{Re}(1)-\mathrm{O}(21)$ & $97.3(4)$ & $C(223)-\operatorname{Re}(2)-S(2)$ & $94.8(4)$ & $\mathrm{C}(321)-\operatorname{Re}(3)-\operatorname{Re}(1)$ & $157.8(4)$ \\
\hline
\end{tabular}




\begin{tabular}{|c|c|c|c|c|c|}
\hline C(123)-Re(1)-O(21) & $175.2(4)$ & C(222)-Re(2)-S(2) & 176.1(4) & C(322)-Re(3)-Re(1) & $113.9(3)$ \\
\hline $\mathrm{C}(122)-\operatorname{Re}(1)-\mathrm{O}(21)$ & $92.8(3)$ & $\mathrm{O}(31)-\operatorname{Re}(2)-\mathrm{S}(2)$ & 80.03(19) & C(323)-Re(3)-Re(1) & $84.6(3)$ \\
\hline N(13)-Re(1)-O(21) & 81.2(3) & N(23)-Re(2)-S(2) & $78.2(2)$ & $\mathrm{N}(33)-\operatorname{Re}(3)-\operatorname{Re}(1)$ & $66.7(2)$ \\
\hline C(121)-Re(1)-S(1) & $177.9(3)$ & $\mathrm{C}(221)-\operatorname{Re}(2)-\operatorname{Re}(1)$ & $113.2(4)$ & $\mathrm{O}(11)-\operatorname{Re}(3)-\operatorname{Re}(1)$ & 19.19(17) \\
\hline C(123)-Re(1)-S(1) & $94.3(4)$ & $\mathrm{C}(223)-\operatorname{Re}(2)-\operatorname{Re}(1)$ & $151.2(4)$ & S(3)-Re(3)-Re(1) & $88.75(7)$ \\
\hline C(122)-Re(1)-S(1) & $93.7(3)$ & $C(222)-\operatorname{Re}(2)-\operatorname{Re}(1)$ & $112.3(4)$ & C(321)-Re(3)-Re(2) & $104.8(4)$ \\
\hline $\mathrm{N}(13)-\operatorname{Re}(1)-\mathrm{S}(1)$ & $78.8(2)$ & $\mathrm{O}(31)-\operatorname{Re}(2)-\operatorname{Re}(1)$ & 64.18(17) & $\mathrm{C}(322)-\operatorname{Re}(3)-\operatorname{Re}(2)$ & $155.2(3)$ \\
\hline $\mathrm{O}(21)-\operatorname{Re}(1)-\mathrm{S}(1)$ & $80.88(18)$ & $\mathrm{N}(23)-\operatorname{Re}(2)-\operatorname{Re}(1)$ & $20.5(2)$ & C(323)-Re(3)-Re(2) & $113.5(3)$ \\
\hline $\mathrm{C}(121)-\operatorname{Re}(1)-\operatorname{Re}(2)$ & $88.7(3)$ & $S(2)-\operatorname{Re}(2)-\operatorname{Re}(1)$ & $64.48(7)$ & $\mathrm{N}(33)-\operatorname{Re}(3)-\operatorname{Re}(2)$ & $17.5(3)$ \\
\hline C(123)-Re(1)-Re(2) & $165.8(3)$ & $\mathrm{C}(221)-\operatorname{Re}(2)-\operatorname{Re}(3)$ & $168.9(4)$ & $\mathrm{O}(11)-\operatorname{Re}(3)-\operatorname{Re}(2)$ & $71.12(17)$ \\
\hline $\mathrm{C}(122)-\operatorname{Re}(1)-\operatorname{Re}(2)$ & 105.8(3) & C(223)-Re(2)-Re(3) & $102.7(3)$ & S(3)-Re(3)-Re(2) & $63.71(6)$ \\
\hline N(13)-Re(1)-Re(2) & $69.5(2)$ & $C(222)-\operatorname{Re}(2)-\operatorname{Re}(3)$ & $86.3(3)$ & $\operatorname{Re}(1)-\operatorname{Re}(3)-\operatorname{Re}(2)$ & $59.738(7)$ \\
\hline O(21)-Re(1)-Re(2) & $15.87(18)$ & O(31)-Re(2)-Re(3) & 13.94(19) & $\mathrm{O}(321)-\mathrm{C}(321)-\operatorname{Re}(3)$ & 177.4(13) \\
\hline$S(1)-\operatorname{Re}(1)-\operatorname{Re}(2)$ & 89.33(7) & N(23)-Re(2)-Re(3) & $72.9(2)$ & $\mathrm{O}(322)-\mathrm{C}(322)-\operatorname{Re}(3)$ & 175.0(11) \\
\hline C(121)-Re(1)-Re(3) & $113.3(3)$ & S(2)-Re(2)-Re(3) & $90.13(7)$ & O(323)-C(323)-Re(3) & 177.5(11) \\
\hline C(123)-Re(1)-Re(3) & 109.2(3) & $\operatorname{Re}(1)-\operatorname{Re}(2)-\operatorname{Re}(3)$ & $60.086(6)$ & C(31)-S(3)-Re(3) & $98.4(4)$ \\
\hline $\mathrm{C}(122)-\operatorname{Re}(1)-\operatorname{Re}(3)$ & $153.2(3)$ & $\mathrm{O}(221)-\mathrm{C}(221)-\operatorname{Re}(2)$ & 178.1(12) & $\mathrm{C}(31)-\mathrm{N}(31)-\mathrm{C}(311)$ & 128.6(10) \\
\hline N(13)-Re(1)-Re(3) & $17.9(2)$ & $\mathrm{O}(222)-\mathrm{C}(222)-\operatorname{Re}(2)$ & $176.2(12)$ & $\mathrm{C}(31)-\mathrm{N}(32)-\mathrm{N}(33)$ & $120.9(10)$ \\
\hline O(21)-Re(1)-Re(3) & $68.65(16)$ & $\mathrm{O}(223)-\mathrm{C}(223)-\operatorname{Re}(2)$ & 177.1(13) & $\mathrm{C}(31)-\mathrm{N}(32)-\mathrm{C}(34)$ & $128.3(10)$ \\
\hline S(1)-Re(1)-Re(3) & 65.09(7) & $C(21)-S(2)-\operatorname{Re}(2)$ & $99.2(4)$ & N(33)-N(32)-C(34) & $110.1(9)$ \\
\hline $\operatorname{Re}(2)-\operatorname{Re}(1)-\operatorname{Re}(3)$ & $60.175(7)$ & $\mathrm{C}(21)-\mathrm{N}(21)-\mathrm{C}(211)$ & $127.6(10)$ & $\mathrm{C}(32)-\mathrm{N}(33)-\mathrm{N}(32)$ & 104.6(9) \\
\hline $\mathrm{O}(121)-\mathrm{C}(121)-\mathrm{Re}(1)$ & $176.6(10)$ & $\mathrm{C}(21)-\mathrm{N}(22)-\mathrm{N}(23)$ & $118.4(9)$ & C(32)-N(33)-Re(3) & 137.1(8) \\
\hline $\mathrm{O}(122)-\mathrm{C}(122)-\mathrm{Re}(1)$ & $178.0(10)$ & $\mathrm{C}(21)-\mathrm{N}(22)-\mathrm{C}(24)$ & $129.8(10)$ & N(32)-N(33)-Re(3) & $117.8(7)$ \\
\hline O(123)-C(123)-Re(1) & $179.4(12)$ & N(23)-N(22)-C(24) & $110.8(9)$ & $\mathrm{C}(34)-\mathrm{O}(31)-\mathrm{Re}(2)$ & $132.1(7)$ \\
\hline C(11)-S(1)-Re(1) & $101.6(4)$ & $\mathrm{C}(22)-\mathrm{N}(23)-\mathrm{N}(22)$ & $103.4(9)$ & $\mathrm{N}(32)-\mathrm{C}(31)-\mathrm{N}(31)$ & $117.0(10)$ \\
\hline $\mathrm{C}(14)-\mathrm{O}(11)-\operatorname{Re}(3)$ & $127.0(7)$ & $\mathrm{C}(22)-\mathrm{N}(23)-\operatorname{Re}(2)$ & $136.4(8)$ & $\mathrm{N}(32)-\mathrm{C}(31)-\mathrm{S}(3)$ & $120.8(9)$ \\
\hline $\mathrm{C}(11)-\mathrm{N}(11)-\mathrm{C}(111)$ & $129.7(10)$ & N(22)-N(23)-Re(2) & $117.8(7)$ & $N(31)-C(31)-S(3)$ & $122.2(9)$ \\
\hline $\mathrm{C}(11)-\mathrm{N}(12)-\mathrm{N}(13)$ & $121.8(9)$ & $\mathrm{C}(24)-\mathrm{O}(21)-\operatorname{Re}(1)$ & $129.0(7)$ & N(33)-C(32)-C(33) & $113.6(11)$ \\
\hline $\mathrm{C}(11)-\mathrm{N}(12)-\mathrm{C}(14)$ & $128.8(10)$ & $\mathrm{N}(21)-\mathrm{C}(21)-\mathrm{N}(22)$ & $115.3(9)$ & N(33)-C(32)-C(35) & $121.3(11)$ \\
\hline N(13)-N(12)-C(14) & $108.8(9)$ & $\mathrm{N}(21)-\mathrm{C}(21)-\mathrm{S}(2)$ & $123.6(9)$ & C(33)-C(32)-C(35) & $125.1(12)$ \\
\hline $\mathrm{C}(12)-\mathrm{N}(13)-\mathrm{N}(12)$ & 106.4(9) & $\mathrm{N}(22)-\mathrm{C}(21)-\mathrm{S}(2)$ & $121.2(8)$ & C(34)-C(33)-C(32) & 106.1(11) \\
\hline $\mathrm{C}(12)-\mathrm{N}(13)-\operatorname{Re}(1)$ & $136.4(8)$ & N(23)-C(22)-C(23) & $112.9(10)$ & $\mathrm{O}(31)-\mathrm{C}(34)-\mathrm{C}(33)$ & 133.5(12) \\
\hline N(12)-N(13)-Re(1) & $116.5(7)$ & N(23)-C(22)-C(25) & $121.5(11)$ & $\mathrm{O}(31)-\mathrm{C}(34)-\mathrm{N}(32)$ & $121.0(10)$ \\
\hline $\mathrm{N}(11)-\mathrm{C}(11)-\mathrm{N}(12)$ & $112.6(9)$ & C(23)-C(22)-C(25) & $125.6(11)$ & $\mathrm{C}(33)-\mathrm{C}(34)-\mathrm{N}(32)$ & 105.6(10) \\
\hline $\mathrm{N}(11)-\mathrm{C}(11)-\mathrm{S}(1)$ & 128.7(9) & C(24)-C(23)-C(22) & $107.0(10)$ & $\mathrm{C}(316)-\mathrm{C}(311)-\mathrm{C}(312)$ & 118.0(12) \\
\hline $\mathrm{N}(12)-\mathrm{C}(11)-\mathrm{S}(1)$ & $118.7(8)$ & $\mathrm{O}(21)-\mathrm{C}(24)-\mathrm{C}(23)$ & $136.5(12)$ & $\mathrm{C}(316)-\mathrm{C}(311)-\mathrm{N}(31)$ & $119.0(10)$ \\
\hline$N(13)-C(12)-C(13)$ & $112.9(10)$ & $\mathrm{O}(21)-\mathrm{C}(24)-\mathrm{N}(22)$ & $117.8(11)$ & $\mathrm{C}(312)-\mathrm{C}(311)-\mathrm{N}(31)$ & $122.7(12)$ \\
\hline
\end{tabular}


Table S4. Bond lengths $[\AA]$ and angles $\left[{ }^{\circ}\right]$ in 2 c.

\begin{tabular}{|c|c|c|c|c|c|}
\hline $\operatorname{Re}(1)-\mathrm{C}(123)$ & $1.906(16)$ & $\mathrm{C}(11)-\mathrm{N}(12)$ & $1.360(16)$ & $\mathrm{C}(115)-\mathrm{C}(116)$ & $1.41(2)$ \\
\hline $\operatorname{Re}(1)-C(121)$ & $1.941(17)$ & N(12)-C(14) & $1.368(16)$ & $\mathrm{O}(121)-\mathrm{C}(121)$ & $1.133(16)$ \\
\hline $\operatorname{Re}(1)-\mathrm{C}(122)$ & 1.959(19) & $\mathrm{N}(12)-\mathrm{N}(13)$ & $1.429(14)$ & $\mathrm{O}(122)-\mathrm{C}(122)$ & $1.127(18)$ \\
\hline $\operatorname{Re}(1)-N(13)$ & $2.162(10)$ & $\mathrm{C}(12)-\mathrm{N}(13)$ & $1.302(15)$ & O(123)-C(123) & $1.129(17)$ \\
\hline $\operatorname{Re}(1)-\mathrm{O}(21)$ & $2.190(9)$ & C(12)-C(13) & $1.403(19)$ & C(211)-C(216) & $1.32(2)$ \\
\hline $\operatorname{Re}(1)-S(1)$ & $2.479(4)$ & C(12)-C(15) & 1.497(18) & C(211)-C(212) & $1.42(2)$ \\
\hline $\operatorname{Re}(1)-\operatorname{Re}(2)$ & $7.4615(10)$ & C(22)-N(23) & $1.352(16)$ & C(212)-C(213) & $1.339(18)$ \\
\hline $\operatorname{Re}(1)-\operatorname{Re}(3)$ & $7.4717(10)$ & C(22)-C(25) & 1.495(17) & C(213)-C(214) & $1.34(2)$ \\
\hline$S(1)-C(11)$ & $1.674(15)$ & C(23)-C(24) & $1.353(17)$ & C(214)-C(215) & $1.32(2)$ \\
\hline $\operatorname{Re}(2)-C(223)$ & $1.882(17)$ & O(31)-C(34) & $1.299(14)$ & C(215)-C(216) & $1.34(2)$ \\
\hline $\operatorname{Re}(2)-C(221)$ & $1.90(2)$ & $\mathrm{N}(31)-\mathrm{C}(31)$ & $1.313(15)$ & $\mathrm{O}(221)-\mathrm{C}(221)$ & $1.176(17)$ \\
\hline $\operatorname{Re}(2)-C(222)$ & $1.935(17)$ & $\mathrm{N}(31)-\mathrm{C}(311)$ & $1.461(16)$ & $\mathrm{O}(222)-\mathrm{C}(222)$ & $1.136(17)$ \\
\hline $\operatorname{Re}(2)-\mathrm{N}(23)$ & $2.146(11)$ & $\mathrm{C}(31)-\mathrm{N}(32)$ & $1.352(15)$ & $\mathrm{O}(223)-\mathrm{C}(223)$ & $1.151(16)$ \\
\hline $\operatorname{Re}(2)-O(31)$ & $2.192(9)$ & N(32)-N(33) & 1.393(13) & C(311)-C(312) & $1.37(2)$ \\
\hline $\operatorname{Re}(2)-S(2)$ & $2.450(4)$ & N(32)-C(34) & $1.401(16)$ & C(311)-C(316) & $1.43(2)$ \\
\hline $\operatorname{Re}(2)-\operatorname{Re}(3)$ & 7.4332(9) & $\mathrm{C}(32)-\mathrm{N}(33)$ & $1.314(15)$ & C(312)-C(313) & $1.46(2)$ \\
\hline$S(2)-C(21)$ & $1.709(14)$ & C(32)-C(33) & $1.404(18)$ & C(313)-C(314) & $1.40(2)$ \\
\hline $\operatorname{Re}(3)-C(323)$ & $1.88(2)$ & C(32)-C(35) & 1.541(17) & C(314)-C(315) & $1.41(2)$ \\
\hline $\operatorname{Re}(3)-C(322)$ & $1.905(19)$ & C(33)-C(34) & $1.319(17)$ & C(315)-C(316) & $1.41(2)$ \\
\hline $\operatorname{Re}(3)-\mathrm{C}(321)$ & $1.963(19)$ & O(321)-C(321) & $1.168(18)$ & C(13)-C(14) & $1.357(17)$ \\
\hline $\operatorname{Re}(3)-N(33)$ & $2.168(11)$ & $\mathrm{O}(322)-\mathrm{C}(322)$ & $1.12(2)$ & $\mathrm{O}(21)-\mathrm{C}(24)$ & $1.306(16)$ \\
\hline $\operatorname{Re}(3)-O(11)$ & $2.197(10)$ & $\mathrm{O}(323)-\mathrm{C}(323)$ & $1.137(19)$ & $\mathrm{N}(21)-\mathrm{C}(21)$ & $1.326(16)$ \\
\hline $\operatorname{Re}(3)-S(3)$ & $2.470(4)$ & C(111)-C(112) & $1.33(2)$ & $N(21)-C(211)$ & $1.436(17)$ \\
\hline S(3)-C(31) & $1.689(14)$ & C(111)-C(116) & $1.40(2)$ & $\mathrm{C}(21)-\mathrm{N}(22)$ & $1.344(15)$ \\
\hline $\mathrm{O}(11)-\mathrm{C}(14)$ & $1.259(14)$ & C(112)-C(113) & $1.39(2)$ & N(22)-N(23) & $1.430(13)$ \\
\hline N(11)-C(11) & $1.326(16)$ & C(113)-C(114) & $1.34(2)$ & $\mathrm{N}(22)-\mathrm{C}(24)$ & $1.432(17)$ \\
\hline $\mathrm{N}(11)-\mathrm{C}(111)$ & $1.453(18)$ & $C(114)-C(115)$ & $1.32(2)$ & $C(22)-C(23)$ & $1.323(19)$ \\
\hline C(123)-Re(1)-C(121) & $89.8(7)$ & $\mathrm{C}(323)-\mathrm{Re}(3)-\mathrm{O}(11)$ & 175.8(6) & N(23)-C(22)-C(25) & $120.9(14)$ \\
\hline C(123)-Re(1)-C(122) & $86.1(7)$ & $\mathrm{C}(322)-\mathrm{Re}(3)-\mathrm{O}(11)$ & $91.1(7)$ & C(22)-N(23)-N(22) & $102.5(10)$ \\
\hline C(121)-Re(1)-C(122) & $86.2(7)$ & C(321)-Re(3)-O(11) & $95.9(6)$ & C(22)-N(23)-Re(2) & 138.2(9) \\
\hline C(123)-Re(1)-N(13) & $97.3(6)$ & N(33)-Re(3)-O(11) & 79.3(4) & $\mathrm{N}(22)-\mathrm{N}(23)-\operatorname{Re}(2)$ & 118.7(8) \\
\hline C(121)-Re(1)-N(13) & 99.2(5) & C(323)-Re(3)-S(3) & 94.1(5) & $C(22)-C(23)-C(24)$ & 109.7(14) \\
\hline C(122)-Re(1)-N(13) & 173.6(5) & C(322)-Re(3)-S(3) & 93.3(5) & $\mathrm{O}(21)-\mathrm{C}(24)-\mathrm{C}(23)$ & $139.7(14)$ \\
\hline $\mathrm{C}(123)-\mathrm{Re}(1)-\mathrm{O}(21)$ & 174.3(6) & C(321)-Re(3)-S(3) & 177.7(5) & $\mathrm{O}(21)-\mathrm{C}(24)-\mathrm{N}(22)$ & $115.3(11)$ \\
\hline $\mathrm{C}(121)-\mathrm{Re}(1)-\mathrm{O}(21)$ & $95.0(5)$ & N(33)-Re(3)-S(3) & $78.6(3)$ & $\mathrm{C}(23)-\mathrm{C}(24)-\mathrm{N}(22)$ & 104.9(13) \\
\hline $\mathrm{C}(122)-\mathrm{Re}(1)-\mathrm{O}(21)$ & $97.2(5)$ & O(11)-Re(3)-S(3) & $81.8(3)$ & C(34)-O(31)-Re(2) & $132.8(8)$ \\
\hline N(13)-Re(1)-O(21) & $78.9(4)$ & C(31)-S(3)-Re(3) & $98.7(5)$ & C(31)-N(31)-C(311) & 134.3(13) \\
\hline C(123)-Re(1)-S(1) & $95.6(5)$ & C(14)-O(11)-Re(3) & 132.4(8) & $\mathrm{N}(31)-\mathrm{C}(31)-\mathrm{N}(32)$ & $114.7(12)$ \\
\hline C(121)-Re(1)-S(1) & 174.4(5) & $\mathrm{C}(11)-\mathrm{N}(11)-\mathrm{C}(111)$ & $129.9(13)$ & N(31)-C(31)-S(3) & $124.2(11)$ \\
\hline $\mathrm{C}(122)-\operatorname{Re}(1)-\mathrm{S}(1)$ & $95.5(5)$ & $\mathrm{N}(11)-\mathrm{C}(11)-\mathrm{N}(12)$ & $114.4(13)$ & N(32)-C(31)-S(3) & 121.1(10) \\
\hline $\mathrm{N}(13)-\operatorname{Re}(1)-\mathrm{S}(1)$ & $78.9(3)$ & N(11)-C(11)-S(1) & $122.7(11)$ & C(31)-N(32)-N(33) & $120.4(10)$ \\
\hline $\mathrm{O}(21)-\operatorname{Re}(1)-\mathrm{S}(1)$ & $79.6(2)$ & N(12)-C(11)-S(1) & $122.7(11)$ & C(31)-N(32)-C(34) & $131.0(11)$ \\
\hline C(11)-S(1)-Re(1) & $98.7(5)$ & $\mathrm{C}(11)-\mathrm{N}(12)-\mathrm{C}(14)$ & 129.6(12) & N(33)-N(32)-C(34) & 108.1(9) \\
\hline C(223)-Re(2)-C(221) & $89.4(6)$ & C(11)-N(12)-N(13) & $118.7(11)$ & N(33)-C(32)-C(33) & $112.7(12)$ \\
\hline C(223)-Re(2)-C(222) & $86.9(7)$ & C(14)-N(12)-N(13) & $110.7(10)$ & $\mathrm{N}(33)-\mathrm{C}(32)-\mathrm{C}(35)$ & $121.6(12)$ \\
\hline C(221)-Re(2)-C(222) & $88.2(6)$ & $\mathrm{N}(13)-\mathrm{C}(12)-\mathrm{C}(13)$ & 111.1(12) & C(33)-C(32)-C(35) & $125.7(12)$ \\
\hline C(223)-Re(2)-N(23) & $98.0(5)$ & N(13)-C(12)-C(15) & $122.3(13)$ & C(32)-N(33)-N(32) & 104.8(10) \\
\hline C(221)-Re(2)-N(23) & 100.1(6) & C(13)-C(12)-C(15) & 126.5(13) & C(32)-N(33)-Re(3) & $136.4(10)$ \\
\hline C(222)-Re(2)-N(23) & $170.4(5)$ & $\mathrm{C}(12)-\mathrm{N}(13)-\mathrm{N}(12)$ & 104.5(10) & $\mathrm{N}(32)-\mathrm{N}(33)-\operatorname{Re}(3)$ & $116.2(7)$ \\
\hline $\mathrm{C}(223)-\mathrm{Re}(2)-\mathrm{O}(31)$ & 176.0(6) & $\mathrm{C}(12)-\mathrm{N}(13)-\operatorname{Re}(1)$ & $137.7(10)$ & C(34)-C(33)-C(32) & $105.4(12)$ \\
\hline $\mathrm{C}(221)-\mathrm{Re}(2)-\mathrm{O}(31)$ & $94.2(5)$ & $\mathrm{N}(12)-\mathrm{N}(13)-\operatorname{Re}(1)$ & $117.2(7)$ & $O(31)-C(34)-C(33)$ & 133.3(14) \\
\hline $\mathrm{C}(222)-\mathrm{Re}(2)-\mathrm{O}(31)$ & $91.7(5)$ & $C(14)-C(13)-C(12)$ & 108.5(13) & O(31)-C(34)-N(32) & $117.7(11)$ \\
\hline $\mathrm{N}(23)-\mathrm{Re}(2)-\mathrm{O}(31)$ & $82.9(4)$ & $\mathrm{O}(11)-\mathrm{C}(14)-\mathrm{C}(13)$ & 135.1(14) & C(33)-C(34)-N(32) & $108.8(12)$ \\
\hline C(223)-Re(2)-S(2) & 95.9(5) & $\mathrm{O}(11)-\mathrm{C}(14)-\mathrm{N}(12)$ & 119.6(12) & $\mathrm{O}(121)-\mathrm{C}(121)-\operatorname{Re}(1)$ & $176.5(15)$ \\
\hline C(221)-Re(2)-S(2) & 174.6(4) & $\mathrm{C}(13)-\mathrm{C}(14)-\mathrm{N}(12)$ & 105.2(12) & $\mathrm{O}(122)-\mathrm{C}(122)-\operatorname{Re}(1)$ & $175.7(15)$ \\
\hline C(222)-Re(2)-S(2) & $93.2(4)$ & C(24)-O(21)-Re(1) & $126.6(8)$ & $\mathrm{O}(123)-\mathrm{C}(123)-\operatorname{Re}(1)$ & 176.9(16) \\
\hline $\mathrm{N}(23)-\operatorname{Re}(2)-\mathrm{S}(2)$ & 78.1(3) & $\mathrm{C}(21)-\mathrm{N}(21)-\mathrm{C}(211)$ & 134.8(13) & $C(216)-C(211)-C(212)$ & 117.1(14) \\
\hline
\end{tabular}




\begin{tabular}{ll|ll|ll}
$\mathrm{O}(31)-\operatorname{Re}(2)-\mathrm{S}(2)$ & $80.5(2)$ & $\mathrm{N}(21)-\mathrm{C}(21)-\mathrm{N}(22)$ & $116.5(12)$ & $\mathrm{C}(216)-\mathrm{C}(211)-\mathrm{N}(21)$ & $127.6(16)$ \\
$\mathrm{C}(21)-\mathrm{S}(2)-\operatorname{Re}(2)$ & $99.0(5)$ & $\mathrm{N}(21)-\mathrm{C}(21)-\mathrm{S}(2)$ & $122.3(11)$ & $\mathrm{C}(212)-\mathrm{C}(211)-\mathrm{N}(21)$ & $115.3(14)$ \\
$\mathrm{C}(323)-\operatorname{Re}(3)-\mathrm{C}(322)$ & $89.0(9)$ & $\mathrm{N}(22)-\mathrm{C}(21)-\mathrm{S}(2)$ & $121.3(11)$ & $\mathrm{O}(221)-\mathrm{C}(221)-\operatorname{Re}(2)$ & $178.9(13)$ \\
$\mathrm{C}(323)-\operatorname{Re}(3)-\mathrm{C}(321)$ & $88.2(7)$ & $\mathrm{C}(21)-\mathrm{N}(22)-\mathrm{N}(23)$ & $117.9(12)$ & $\mathrm{O}(222)-\mathrm{C}(222)-\operatorname{Re}(2)$ & $176.3(15)$ \\
$\mathrm{C}(322)-\operatorname{Re}(3)-\mathrm{C}(321)$ & $86.7(7)$ & $\mathrm{C}(21)-\mathrm{N}(22)-\mathrm{C}(24)$ & $132.5(12)$ & $\mathrm{O}(223)-\mathrm{C}(223)-\operatorname{Re}(2)$ & $179.3(17)$ \\
$\mathrm{C}(323)-\operatorname{Re}(3)-\mathrm{N}(33)$ & $100.0(6)$ & $\mathrm{N}(23)-\mathrm{N}(22)-\mathrm{C}(24)$ & $108.9(10)$ & $\mathrm{O}(321)-\mathrm{C}(321)-\operatorname{Re}(3)$ & $177.8(16)$ \\
$\mathrm{C}(322)-\operatorname{Re}(3)-\mathrm{N}(33)$ & $168.2(6)$ & $\mathrm{C}(23)-\mathrm{C}(22)-\mathrm{N}(23)$ & $114.0(12)$ & $\mathrm{O}(322)-\mathrm{C}(322)-\operatorname{Re}(3)$ & $172(2)$ \\
$\mathrm{C}(321)-\operatorname{Re}(3)-\mathrm{N}(33)$ & $101.1(5)$ & $\mathrm{C}(23)-\mathrm{C}(22)-\mathrm{C}(25)$ & $124.9(13)$ & $\mathrm{O}(323)-\mathrm{C}(323)-\operatorname{Re}(3)$ & $176.6(17)$ \\
\hline
\end{tabular}


Table S5. Hydrogen bonds in the trimeric complexes $\left[\operatorname{Re}_{3}\left(\mathrm{pyz}^{\mathrm{R}}\right)_{3}(\mathrm{CO})_{9}\right]\left(\AA\right.$ and $\left.{ }^{\circ}\right)$.

\begin{tabular}{lllll}
\hline $\mathrm{D}-\mathrm{H} \ldots \mathrm{A}$ & $\mathrm{d}(\mathrm{D}-\mathrm{H})$ & $\mathrm{d}(\mathrm{H} \ldots \mathrm{A})$ & $\mathrm{d}(\mathrm{D} \ldots \mathrm{A})$ & $<(\mathrm{DHA})$ \\
\hline 2b.1/2C $\mathbf{H}_{\mathbf{8}}$ & & & & \\
$\mathrm{N}(11)-\mathrm{H}(11) \ldots \mathrm{O}(11)$ & 0.86 & 1.90 & $2.60(3)$ & 137.2 \\
$\mathrm{~N}(21)-\mathrm{H}(21) \ldots \mathrm{O}(21)$ & 0.86 & 1.99 & $2.67(3)$ & 135.4 \\
$\mathrm{~N}(31)-\mathrm{H}(31) \ldots \mathrm{O}(31)$ & 0.86 & 1.87 & $2.55(4)$ & 135.3 \\
$\mathrm{C}(311)-\mathrm{H}(31 \mathrm{~A}) \ldots \mathrm{O}(223)^{\# 1}$ & 0.96 & 2.60 & $3.22(4)$ & 123.1 \\
$\mathrm{C}(111)-\mathrm{H}(11 \mathrm{~B}) \ldots \mathrm{O}(222)^{\# 1}$ & 0.96 & 2.68 & $3.28(3)$ & 120.9 \\
$\mathrm{C}(211)-\mathrm{H}(21 \mathrm{~B}) \ldots \mathrm{O}(122)^{\# 2}$ & 0.96 & 2.48 & $3.17(4)$ & 129.2 \\
$\mathrm{C}(211)-\mathrm{H}(21 \mathrm{~A}) \ldots \mathrm{O}(323)^{\# 3}$ & 0.96 & 2.56 & $3.41(4)$ & 148.2
\end{tabular}

Symmetry transformations: \#1 -x+1/2,y+1/2,-z+1 \#2-x+1,y,-z+2 \#3-x+1/2,y-1/2,-z+1

\section{$2 c .2 \mathrm{C}_{7} \mathbf{H}_{8}$}

$\begin{array}{lllll}\mathrm{N}(11)-\mathrm{H}(11) \ldots \mathrm{O}(11) & 0.86 & 1.85 & 2.606(10) & 146.5\end{array}$

$\mathrm{N}(21)-\mathrm{H}(21) \ldots \mathrm{O}(21) \quad 0.86 \quad 1.90 \quad 2.617(10) \quad 140.5$

$\mathrm{N}(31)-\mathrm{H}(31) \ldots \mathrm{O}(31) \quad 0.86 \quad 1.91 \quad 2.628(11) \quad 140.3$

$\mathrm{C}(35)-\mathrm{H}(35 \mathrm{~B}) \ldots \mathrm{O}(223)^{\# 1} \quad 0.96 \quad 2.87 \quad 3.375(13) \quad 114.7$

Symmetry transformations: \#1 -x+2,y+1/2,-z+1/2

2c

$\begin{array}{lllll}\mathrm{N}(11)-\mathrm{H}(11) \ldots \mathrm{O}(11) & 0.86 & 1.85 & 2.576(14) & 141.5\end{array}$

$\mathrm{N}(21)-\mathrm{H}(21) \ldots \mathrm{O}(21) \quad 0.86 \quad 1.92 \quad 2.660(13) \quad 143.3$

$\mathrm{N}(31)-\mathrm{H}(31) \ldots \mathrm{O}(31) \quad 0.86 \quad 1.84 \quad 2.596(13) \quad 145.2$

$\mathrm{C}(315)-\mathrm{H}(315) \ldots \mathrm{O}(223)^{\# 1} \quad 0.93 \quad 2.64 \quad 3.49(2) \quad 153.6$

$\mathrm{C}(213)-\mathrm{H}(213) \ldots \mathrm{O}(223)^{\# 2} \quad 0.93 \quad 2.68 \quad 3.36(2) \quad 131.0$

$\mathrm{C}(15)-\mathrm{H}(15 \mathrm{~A}) \ldots \mathrm{O}(221)^{\# 3} \quad 0.96 \quad 2.63 \quad 3.36(2) \quad 133.3$

Symmetry transformations: \#1 -x+1/2,y-1/2,-z+1/2 \#2 -x+1,-y,-z+1 \#3 x+1/2,-y+1/2,z+1/2 


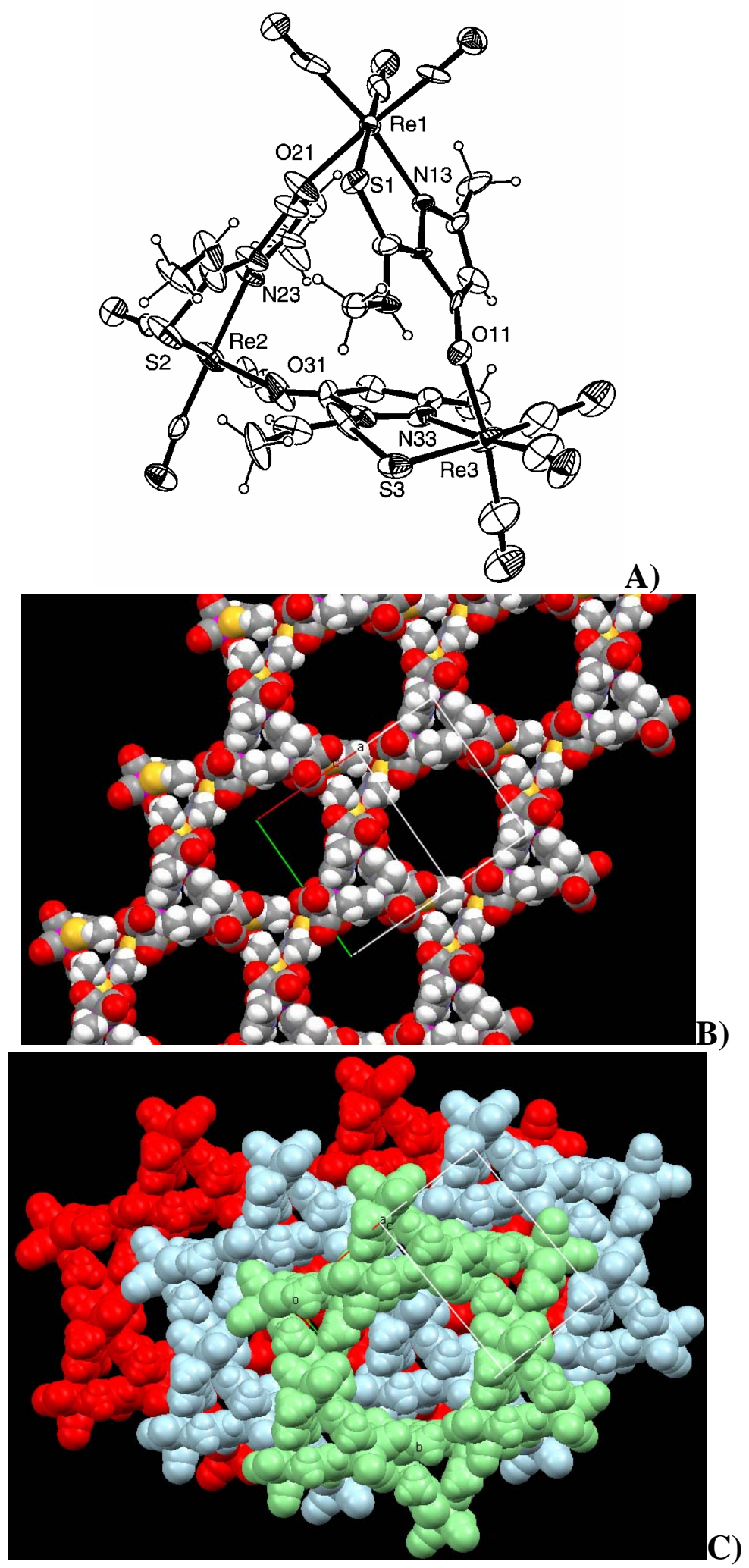

Figure S1. Ellipsoid representation (30\% probability) of $\mathbf{2 b} \cdot \mathbf{1} / \mathbf{2} \mathbf{C}_{\mathbf{7}} \mathbf{H}_{\mathbf{8}}(\mathbf{A})$, and space-filling diagrams showing the formation of sheets with hexagonal holes parallel to the $(-210)$ crystallographic plane (B), and the relative positions of these sheets (C). 


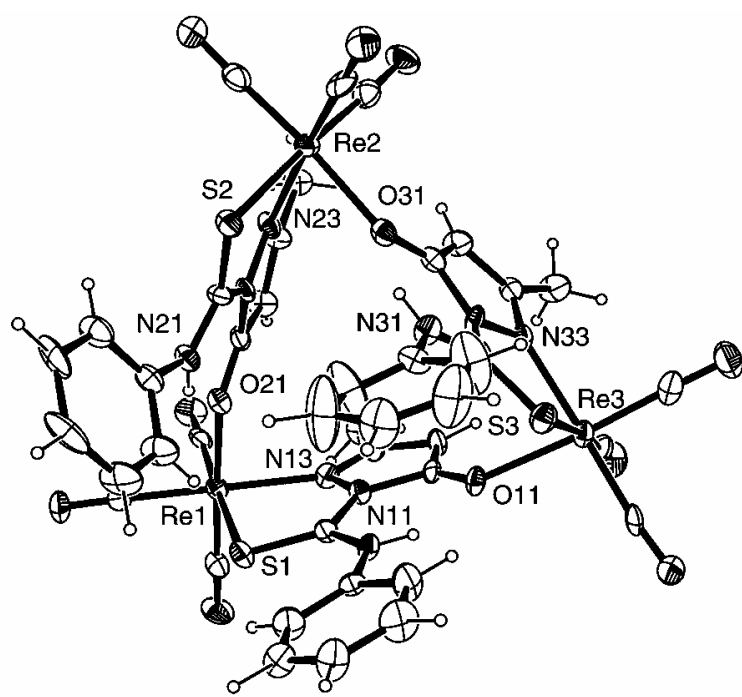

A)
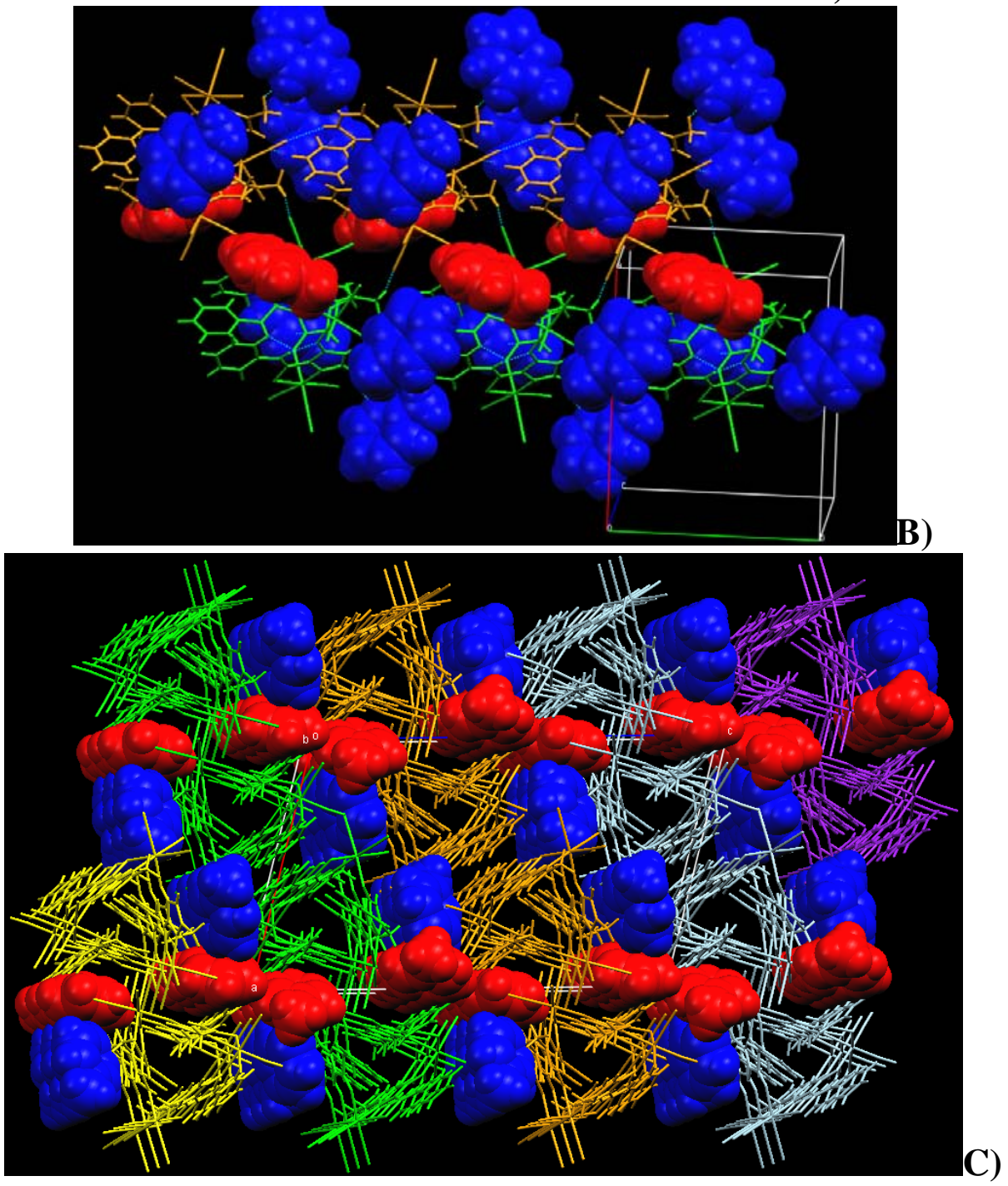

Figure S2. Ellipsoid representation (30\% probability) of $2 \mathbf{c} .2 \mathbf{C}_{\mathbf{7}} \mathbf{H}_{\mathbf{8}}(\mathbf{A})$, and space-filling diagrams showing the packing of the trimer units and two types of toluene molecule (one depicted in red, the other in blue) $(\mathbf{B}, \mathbf{C})$. 

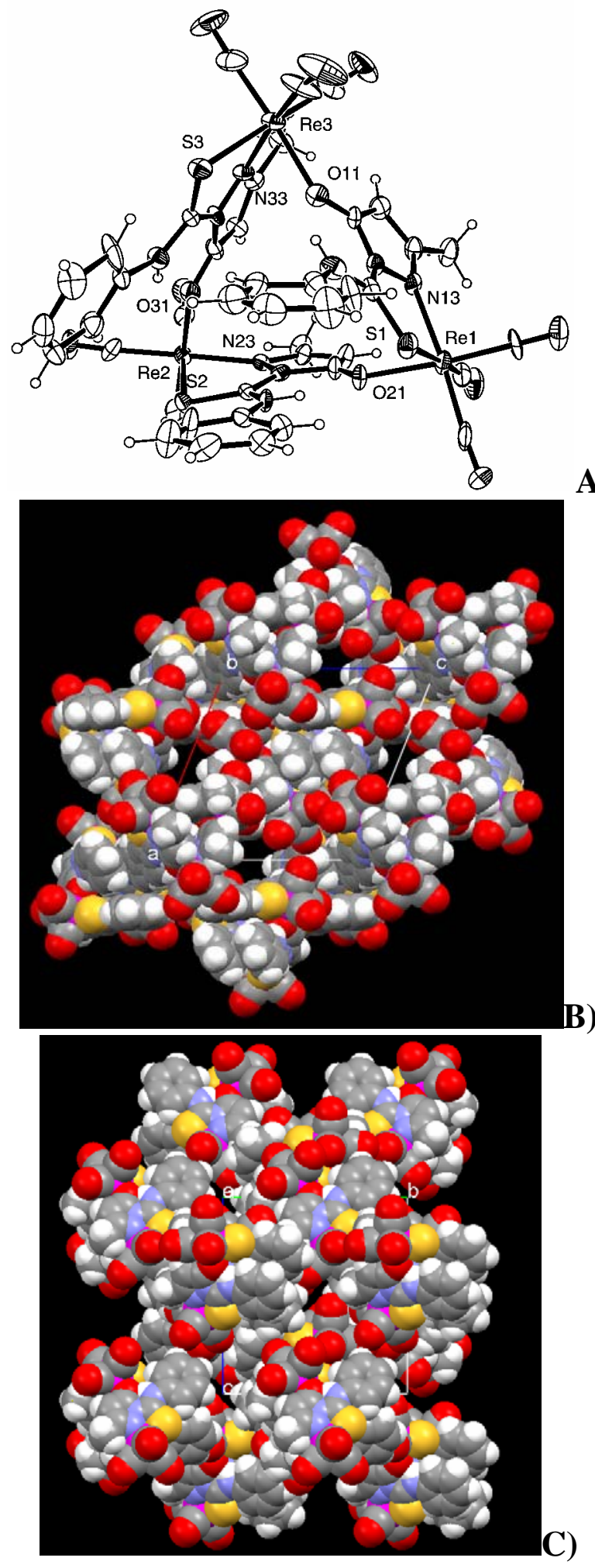

Figure S3. Ellipsoid representation (30\% probability) of $\mathbf{2 c}(\mathbf{A})$, and spacefilling diagrams looking down the $b(\mathbf{B})$ and $a(\mathbf{C})$ axes, showing the channels running along the $b$ axis. 


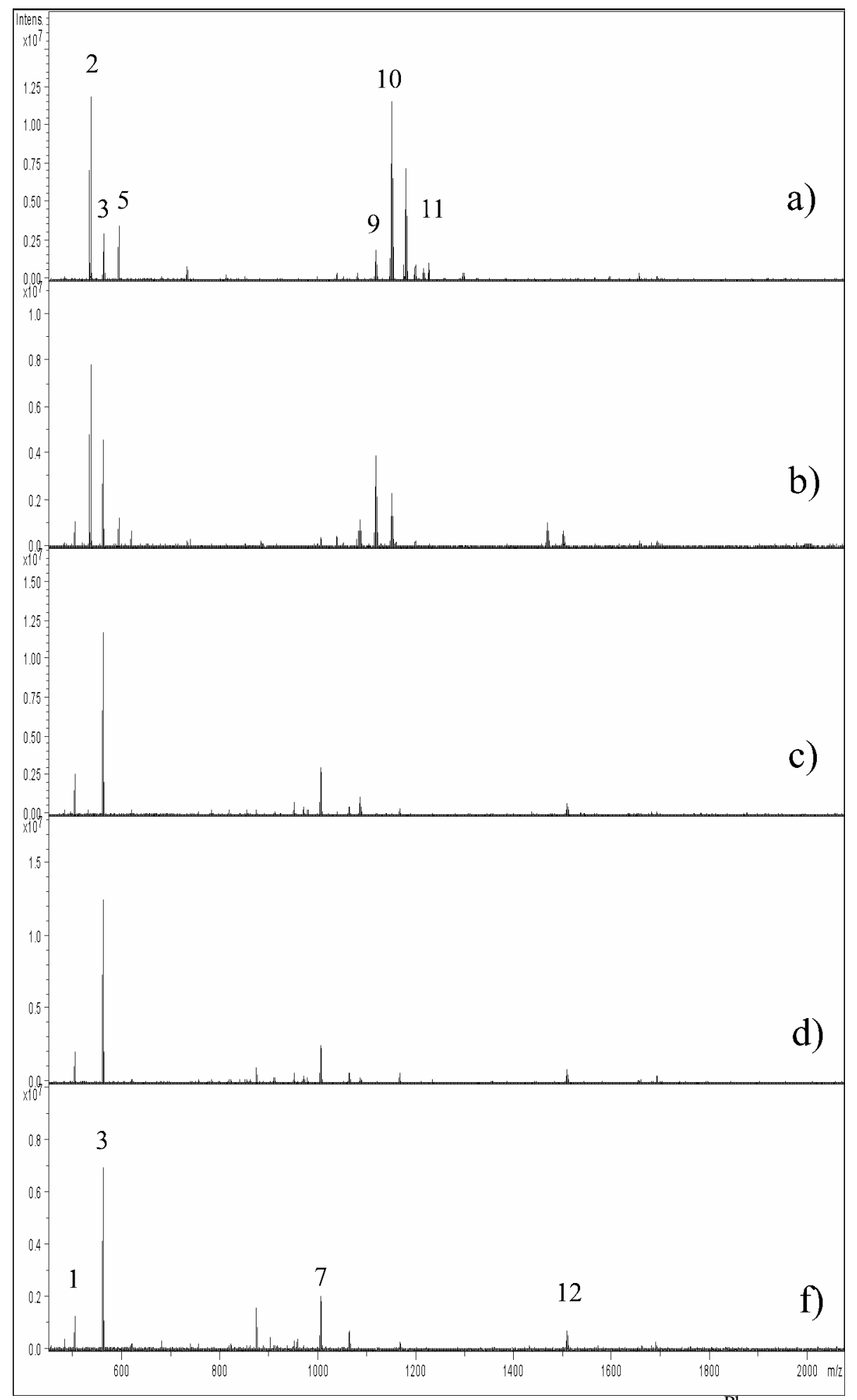

Figure S4. ESI-FTICR-MS spectra of the reaction of $\left[\mathrm{ReBr}(\mathrm{CO})_{5}\right]$ and $\mathrm{H}_{2} \mathrm{~L}^{\mathrm{Ph}}$ in the $\mathrm{m} / \mathrm{z}$ range of 490-2100 (a) at 0 (a), $0.25 \mathrm{~h}$ (b), $2.5 \mathrm{~h} \mathrm{(c),5.5} \mathrm{h} \mathrm{(d)} \mathrm{and} 15 \mathrm{~h}$ (e) after the reaction mixture began to reflux. Peak assignments are listed in Table 1. 